\title{
2540. Fault detection and diagnosis of rotating machinery using modified particle filter
}

\author{
Ke Li ${ }^{1}$, Yiya Liu ${ }^{2}$, Jingjing $\mathrm{Wu}^{3}$, Lei Su${ }^{4}$, Peng Chen ${ }^{5}$ \\ 1,2,3,4 Jiangsu Key Laboratory of Advanced Food Manufacturing Equipment and Technology, \\ Jiangnan University, $1800 \mathrm{Li}$ Hu Avenue, Wuxi, Jiangsu, 214122, China \\ ${ }^{1,5}$ Graduate School of Bioresources, Mie University, Mie, 514-8507, Japan \\ ${ }^{3}$ Corresponding author \\ E-mail: 1ike@jiangnan.edu.cn, ${ }^{2} 2419146862 @ q q . c o m,{ }^{3}$ wjjlady720@jiangnan.edu.cn, \\ 43002965@qq.com, ${ }^{5}$ chen@bio.mie-u.ac.jp
}

Received 5 December 2016; received in revised form 3 May 2017; accepted 4 May 2017 DOI https://doi.org/10.21595/jve.2017.18078

Abstract. In order to effectively monitor condition and detect fault types of high nonlinear system, and extract the features of system state under strong noise background, this paper proposes a novel fault detection and diagnosis (FDD) method based on modified particle filter (PF). The artificial neural network is incorporated in PF for adaptively adjusting weight of particle. In the modified $\mathrm{PF}$, the large weight particles are split into several small weight particles, the particles with smaller weight is adjusted by using artificial neural network. By which the particles in the low probability density region are adjusted to the high probability density region, and the problem of particle leanness is solved effectively. Moreover, this paper also uses time-varying auto regressive (TVAR) and Akaike information criterion (AIC) methods to establish state space model for state estimation. Finally, the proposed method is implemented for fault diagnosis on a roller bearing. Good results are obtained, and the bearing faults, such as the outer race, the inner race and the roller element defects, have been effectively discriminated.

Keywords: fault detection and diagnosis, particle filter, time-varying auto regressive, state estimation.

\section{Introduction}

Fault detection and diagnosis (FDD) have vast importance to improve production efficiency, ensure production safety, and reduce maintenance costs of plant. FDD based on vibration analysis techniques are most common and effective method, which are widely used in mechanical fault diagnosis and condition monitoring. However, due to the complexity of the plant structure and the noise interference in the work locale, the vibration signals measured for mechanical fault diagnosis and condition monitoring are highly nonlinear, and often contain stronger noise components [1-3]. The information that can reflect mechanical condition is buried via noise components. In these cases, mechanical status cannot be effectively detected, and even may lead to misrecognition. Therefore, how to effectively remove the noise and extract the features of the fault signals are very important for mechanical fault diagnosis and condition monitoring.

Many studies based on vibration signal processing technology for mechanical fault diagnosis purpose have been carried out. Signal filtering techniques based on Fourier transform have been the dominating signal processing tool for condition diagnosis [4-9], and widely applied in engineering practice. Sadly, Fourier transform has some limitations, such as the signal to be processed must satisfy periodicity and stationary requirements. Thus, Fourier transform is not applicable to nonstationary or nonlinear signals, and unable to reveal the intrinsic features of nonstationary and nonlinear signals $[10,11]$. Wavelet Transform (WT) is a power tool for analyzing nonstationary signals, and has become a hot spot in the research of modern signal processing. WT method shows a good picture in the joint time-frequency domain, and clearly describe the change of frequency vary with time. In [12] the discrete WT was used to decompose the vibration signal of the rolling bearing, and spalling failure of rolling bearing was successfully predicted online. In [13] wavelet packet and support vector machine were integrated to detect faults of a rolling bearing. However, the results of signal de-noising largely depend on the 
selection of wavelet basis functions. Only selecting the appropriate basis function, the features of signal can be well to detect. In addition, because of the limited length of the wavelet basis function, the leakage of signal energy is inescapable. Kalman filter is an optimal recursive data processing algorithm, which often be used to estimate system state for linear systems with Gaussian noise. In [14] a Kalman filter based on the methodology of the maximum likelihood estimation was established, by which sensor fault was correctly estimated. Reference [15] used Kalman filter to predict the future state, so as to realize the preventive maintenance of DC motor. In order to extend Kalman filter to the nonlinear system, extended Kalman filter (EKF) [16] and unscented Kalman filter (UKF) [17] were proposed. However, when the system is strongly nonlinear, filtering accuracy of these improved Kalman filter will be greatly reduced, and even lead to output divergence of system filtering.

$\mathrm{PF}$ is recursive Bayesian filtering estimation based on sequential Monte Carlo algorithm. It is a nonlinear filtering method, and provides an effective way to solve the state estimation of nonlinear system under non Gauss noise. In the last decade, PF has been widely applied in many fields, such as tracking problem, mobile robot navigation, mechanical fault diagnosis and machinery life prediction [18-23]. In [19] authors combined PF with $M$-algorithm, by which sensor faults were detected accurately and effectively. In [20] authors proposed a new PF based on genetic operator's algorithm, which called intelligent PF. This method was used for fault diagnosis of a nonlinear system, and got good results. In [21] PF and UKF method were integrated for FDD, and two numerical examples verified the effectiveness of this method.

In the fault diagnosis of rotating machinery, the vibration response is obviously nonlinear due to the changes of stiffness, friction, fault excitation and external excitation and so on. Multiple vibrations are coupled to each other and fault characteristic signal is buried under strong noise background. Although the traditional PF can be used as an effective means to solve the nonlinear problem, but the algorithm still has some problems. The traditional PF suffer from a loss of diversity among particles after resampling and cannot accurately characterize posterior probability density. To solve FDD of high nonlinear system, and extract the features of machinery state under strong noise background, this paper proposes a novel FDD method based on modified PF. The artificial neural network is incorporated in PF for adaptively adjusting weight of particle, by which the particles in the low probability density region are adjusted to the high probability density region, and the problem of particle leanness is solved effectively.

The structure of this paper is organized as follows, Section 2 introduces the theory of the general PF, and expounds the problem in the general PF; In Section 3, the modified PF based on artificial neural network is described detailed, and the example that commonly used in PF testing is presented; In Section 4, the practical example of mechanical fault diagnosis for a bearing is designed to verify the effectiveness of the proposed method, and we also compared the proposed method with the WT technique. Section 5 summarizes and concludes this paper.

\section{Theory of particle filter}

$\mathrm{PF}$ is recursive Bayesian filtering estimation based on Monte Carlo algorithm [24]. The essence of $\mathrm{PF}$ is to approximate the posterior probability density by a set of weighted random samples.

In general, the system of state equation and measurement equation can be expressed as:

$x_{t}=f_{t}\left(x_{t-1}\right)+\omega_{t-1}$

$z_{t}=h\left(x_{t}\right)+\varepsilon_{t}$,

where, $x_{t}$ express the system state variable at time $t$, and $z_{t}$ is the observed value of $x_{t}$ at time $t ; \omega_{t}$ and $\varepsilon_{t}$ indicate system noise and measurement noise, respectively; $f(\cdot)$ : $R^{n_{x}} \times R^{\omega_{x}} \rightarrow R^{n_{x}}$ indicates state transition function, and $h(\cdot): R^{n_{x}} \times R^{\varepsilon_{x}} \rightarrow R^{n_{z}}$ expresses the measurement function.

The recursive Bayesian filtering includes two steps: prediction and updating. 
Prediction:

$$
\begin{aligned}
& p\left(x_{t} \mid z_{1: t-1}\right)=\int p\left(x_{t} \mid x_{t-1}, z_{10: t-1}\right) p\left(x_{t-1} \mid z_{1: t-1}\right) d x_{t-1} \\
& =\int p\left(x_{t} \mid x_{t-1}\right) p\left(x_{t-1} \mid z_{1: t-1}\right) d x_{t-1} .
\end{aligned}
$$

Updating:

$$
p\left(x_{t} \mid z_{1: t}\right)=\frac{p\left(x_{t} \mid z_{1: t-1}\right) p\left(z_{t} \mid x_{t}\right)}{p\left(z_{t} \mid z_{1: t-1}\right)},
$$

where:

$p\left(z_{t} \mid z_{1: t-1}\right)=\int p\left(x_{t} \mid z_{1: t-1}\right) p\left(z_{t} \mid x_{t}\right) d x_{t}$

For non-linear and non-Gaussian systems, it is difficult to obtain posterior probability $p\left(x_{t} \mid z_{1: t}\right)$ by the analytic solution. Therefore, the sequential importance sampling (SIS) method is applied in PF. SIS algorithm transforms integral operation into sum operation of samples, and approximates the state posterior probability density by a set of weighted particles $\left\{x_{0: t}^{i}, \omega_{t}^{i}\right\}_{i=1}^{N}$ that sampled from an importance probability density function. The posterior probability $p\left(x_{0: t} \mid z_{1: t}\right)$ at time $t$ can be approximated by the particles as follows:

$p\left(x_{0: t} \mid z_{1: t}\right)=\sum_{i=1}^{N} \omega_{t}^{i} \delta\left(x_{0: t}-x_{0: t}^{i}\right)$,

where, $N$ is the number of the particles.

The importance probability density function:

$q\left(x_{0: t} \mid z_{1: t}\right)=q\left(x_{t} \mid x_{t-1,}, z_{t}\right) q\left(x_{0: t-1} \mid z_{1: t-1}\right)$.

The weight $\omega_{t}^{i}$ for each particle:

$\omega_{t}^{i}=\frac{p\left(x_{0: t} \mid z_{1: t}\right)}{q\left(x_{0: t} \mid z_{1: t}\right)} \propto \omega_{t-1}^{i} \frac{p\left(z_{t} \mid x_{t}^{i}\right) p\left(x_{t}^{i} \mid x_{t-1}^{i}\right)}{q\left(x_{t}^{i} \mid x_{0: t-1}^{i}, z_{1: t}\right)}$,

where, the symbol $\propto$ indicates to be proportional to.

The normalized weight $\widetilde{\omega}_{t}^{i}$ is:

$\widetilde{\omega}_{t}^{i}=\frac{\omega_{t}^{i}}{\sum_{i=1}^{N} \omega_{t}^{i}}$.

The posterior probability $p\left(x_{0: t} \mid z_{0: t}\right)$ is rewritten as:

$p\left(x_{0: t} \mid z_{1: t}\right)=\sum_{i=1}^{N} \widetilde{\omega}_{t}^{i} \delta\left(x_{0: t}-x_{0: t}^{i}\right)$,

where, $\delta(\cdot)$ is Dirac-delta function.

The state $x_{t}$ can be estimated as: 
$\hat{x}_{t} \approx \sum_{i=1}^{N} \widetilde{\omega}_{t}^{i} x_{t}^{i}$.

In SIS, after a few iterations, most of particles have negligible weights, PF algorithm occurs particle degeneracy [25]. To deal with the particle degeneracy problem, this study adopts multinomial resampling technique, and its procedure is shown in Table 1.

Table 1. Multinomial resampling

\begin{tabular}{l}
\hline Step 1: For $i=1,2, \ldots, N$, draw a sample set $\left\{u_{i}\right\}$ from the uniformly distribution $(0,1]$ \\
\hline Step 2: For $i=1,2, \ldots, N$, find the variable quantity $j(j=1,2, \ldots, N)$ that satisfies \\
$\qquad \sum_{n=1}^{j-1} \omega_{n}<u_{i} \leq \sum_{n=1}^{j} \omega_{n}$. \\
\hline Step 3: Store the $x_{t}^{i}$ as a new particle, and the weight of the new particle $\omega_{t}^{i}=1 / N$ \\
\hline
\end{tabular}

The degree of particle degeneracy can be appraised using the effective sample size $N_{\text {eff }}$ [26]:

$N_{e f f}=\frac{1}{\sum_{i=1}^{N}\left(\widetilde{\omega}_{t}^{i}\right)^{2}}$

From Eq. (12), the smaller value of the effective sample size $N_{e f f}$, the more serious the problem of particle degradation will be. Setting the threshold of effective sample $N_{r}, N_{r}=m \times N, m \in(0$, 1). If $N_{e f f}<N_{r}$ particle degradation is serious, PF system performs resampling. After resampling process, the particles that have small weight are knocked out, and many new particles are obtained by the particles that have big weight, as shown in Fig. 1. The procedure of PF is presented as Table 2 .

Table 2. The procedure of PF

Step 1: Set time $t=0$, for $i=1,2, \ldots, N$, draw the particles $x_{0}^{i}$ from the prior probability density, and all of the particles have same weight $1 / N$

Step 2: For $i=2,3, \ldots, N$, draw the particles $x_{t}^{i}$ from the importance probability density, calculate and normalize the particles weight $\omega_{t}^{i}$ by Eqs. (8) and Eq. (9)

Step 3: Calculate the effective sample size $N_{e f f}$. If $N_{e f f}<N_{r}$, resampling is carried out, the new particle set $\left\{x_{0: t}^{j}\right\}_{j=1}^{N}$ is produced. If $N_{e f f}<N_{r}$, PF go to step 4, directly

Step 4: Estimate the state by Eq. (11)

Step 5: Set $t=t+1$, and obtain the new measurement data, return to step (2)

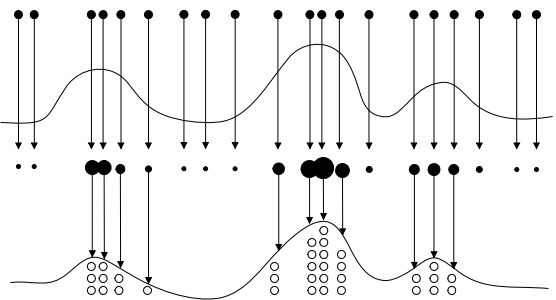

Fig. 1. Illustration of resampling

In resampling procedure, only the particles that have big weight are selected as copy samples, the new particles are replicas of them, and the particles with small weight are eliminated. In most cases, all the resampling operations are focused on a few particles with big weight, the particles lose diversity. This problem is also called particle leanness. The simple method to solve particle leanness 
problem is to increase the number of particle. However, computing burdens of PF will be increased. If computing time of $\mathrm{PF}$ is too long, $\mathrm{PF}$ is difficult to be applied in practical engineering.

For above the problems, this study proposed a novel PF algorithm based on BPNN, which is described in the next section.

\section{Modified particle filter based on NN}

In this section, an improved PF which is named AWPF is presented. The inspiration of this method is from back propagation neural network (BPNN).

\subsection{Brief of BPNN}

BPNN is a kind of multi layer feed-forward network which is trained by the error back propagation algorithm, and consist of input layer, hidden layer and output layer [27]. The gradient descent method is used to adjust the weight and threshold between each layer of BP-NN, to minimize sum of the squared errors. Let input data is $a_{i}(i=1,2, \ldots, N)$, and corresponding teacher data is $x_{i}(i=1,2, \ldots, N), y_{i}$ is actual output. The network error function is as follows:

$E=\frac{1}{2} \sum_{i=1}^{n}\left(x_{i}-y_{i}\right)^{2}$.

The weight adjustment function is as Eq. (15):

$\Delta w_{i j}=-\eta \frac{\partial E}{\partial w_{i j}}$

where, $\eta$ is learning rate of BPNN; $w_{i j}$ indicates the weight between each layer; $\Delta w_{i j}$ expresses modified quantity of the weight.

\subsection{AWPF}

In AWPF algorithm, abruption and adjustment of particle weight are two basic and important operations, which are described as follows.

Particles abruption:

(1) Obtain the particles $x_{t}^{i}, i=0,1, \ldots, N$ from the importance posterior probability density, and calculate the particles weight $\omega_{t}^{i}$ by Eq. (8), and normalize the particles weight $\widetilde{\omega}_{t}^{i}$ by Eq. (9);

(2) Divide all of the particles into large weight particles and small weight particles by the strategy instructed as follows:

$x_{t}^{i} \in \begin{cases}N_{b}, & \widetilde{\omega}_{t}^{i} \geq R, \\ N_{s}, & \widetilde{\omega}_{t}^{i}<R,\end{cases}$

where, $N_{b}$ and $N_{s}$ are large weight and small weight particle sets, respectively; $R$ is a threshold for separating the particles.

(3) Split every large weight particle in set $N_{b}\left\{x_{t}^{i}, \widetilde{\omega}_{t}^{i}\right\}_{i=1}^{p}$ into two new particles, and the new particles weights equal to half of the before.

(4) After step (3) the number of particle has been increased to $N+p$. In order to keep the number of particle unchanged, $p$ particles with the minimum weight in set $N_{s}$ are eliminated.

Weight adjustment:

(1) Sort the particles with the weight values in descending order;

(2) Select $q$ particles with the minimum weight, and adjust their weights by using BPNN. The 
network error function is:

$\varepsilon=\frac{1}{2} \sum_{i=1}^{q}\left(z_{i}-y_{i}\right)^{2}=\frac{1}{2} \sum_{i=1}^{q}\left(e_{k}\right)^{2}$,

where, $q$ is the number of input and output unit of the BPNN; $z_{i}$ is the measurement value, and used as teacher data; $y_{i}$ is output of the BPNN.

Fig. 2 is the BPNN diagram for PF algorithm. In Fig. 2, the input units are the state values of the particle with the minimum weight, and the corresponding weight of the particles are used as the initial weights of BPNN, the output units are the new particle weight values after adjustment, the number of hidden layer is set to eight.

After particles abruption and weight adjustment steps, the particles in the low probability density region are adjusted to the high probability density region, and the particle diversity is increased. The flowchart of AWPF is shown in Fig. 3.

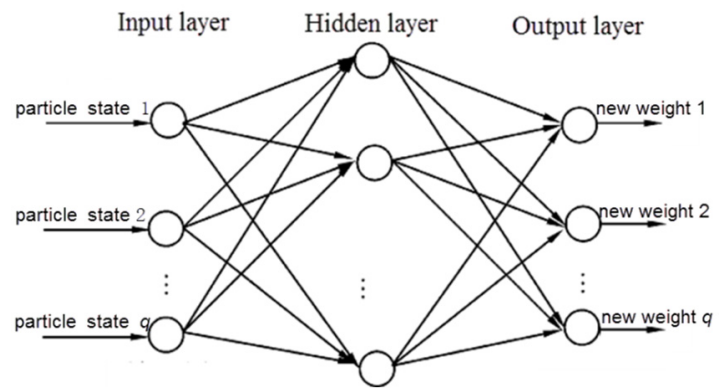

Fig. 2. BPNN diagram for PF algorithm

\subsection{Simulation}

In order to verify the effectiveness of AWPF, an example that commonly used in PF testing is presented, and described as follows:

$x_{t}=0.5 x_{t-1}+\frac{25 x_{t-1}}{1+\left(x_{t-1}\right)^{2}}+8 \cos 1.2(t-1)+\omega_{t-1}$,

$y_{t}=0.05\left(x_{t}\right)^{2}+v_{t}$,

where, $\omega_{t-1}$ is state noise, and $\omega_{t-1} \sim\left(N, \sigma_{w}^{2}\right) ; v_{t}$ is measurement noise, and $v_{t} \sim\left(N, \sigma_{v}^{2}\right)$; in present test, $\sigma_{w}^{2}$ and $\sigma_{v}^{2}$ are 0.1 and 0.01 , respectively; initial probability density function $p\left(x_{o}\right) \sim N(0,2)$; the number of the particle is set to 1000 . This model is strong nonlinear and bimodal.

Root mean square error (RMSE) as shown in Eq. (20) is chosen to estimate the performance of AWPF:

$R M S E=\sqrt{\frac{1}{n} \sum_{i=1}^{n}\left(x_{i}-\hat{x}_{i}\right)^{2}}$,

where $x_{i}$ is the simulation data. $\hat{x}_{i}$ indicates estimate data. $n$ expresses length of the data.

The simulations for the general PF and AWPF are performed on same test condition. Fig. 4 and Fig. 5 show the state estimate results and RMSE curve of the general PF and AWPF, respectively. Comparison of simulation data is listed in Table 3. As shown in Table 3, the mean accuracy of the general PF and AWPF are 0.568 and 0.292 , the available samples of the general PF and AWPF are 192 and 405, respectively. The mean accuracy and the available samples of 
AWPF are greater than the general PF. That is to say AWPF can improve particle diversity and state estimate accuracy.

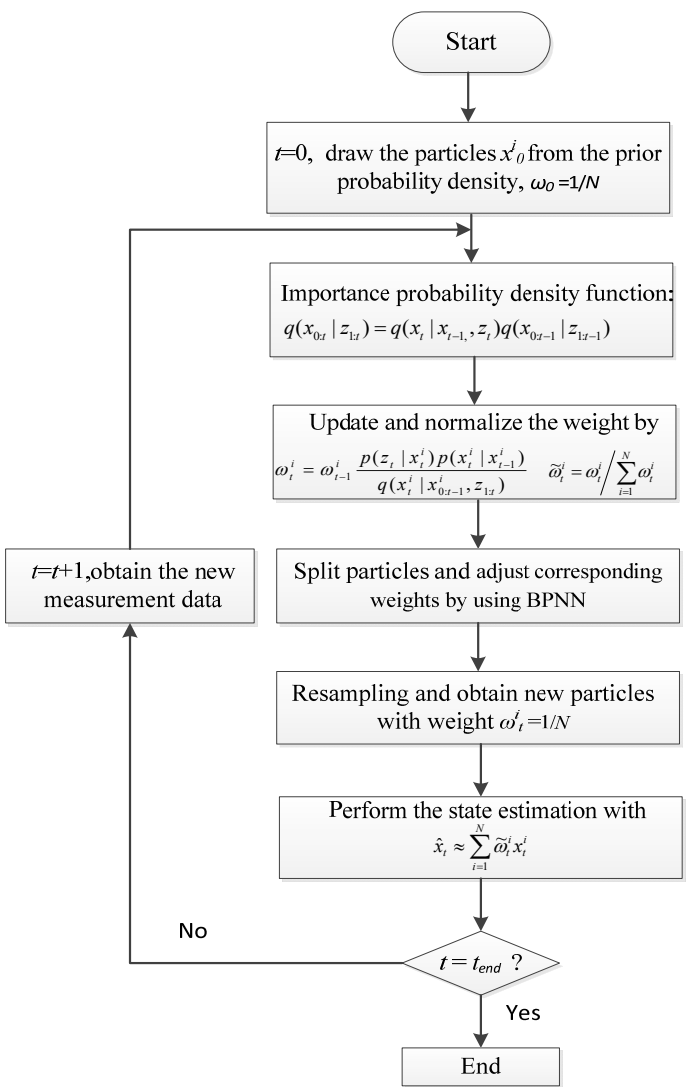

Fig. 3. flowchart of AWPF

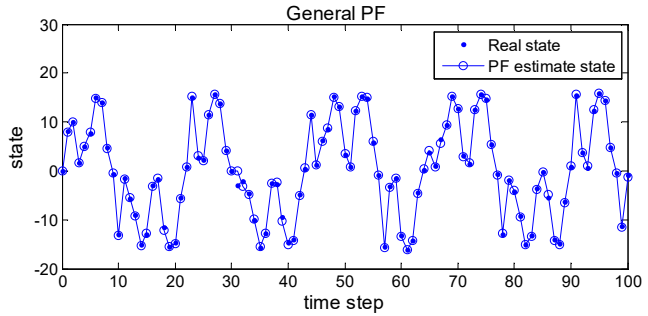

a)

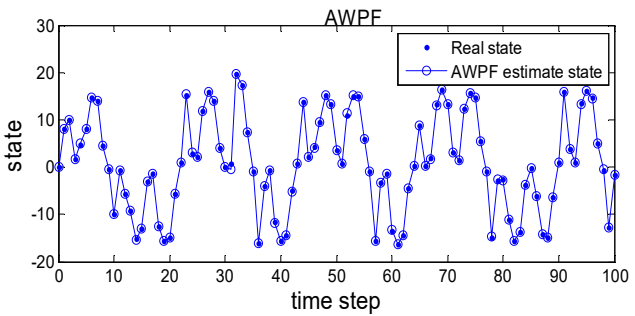

b)

Fig. 4. State estimate results using general PF and AWPF

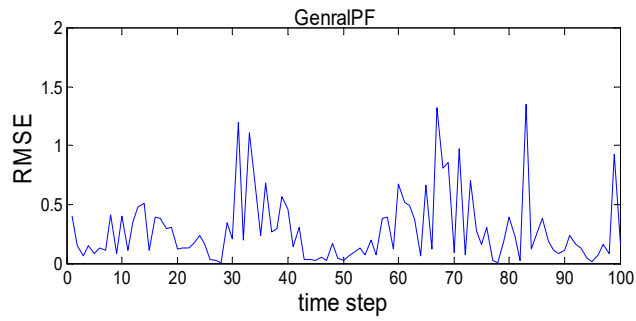

a)

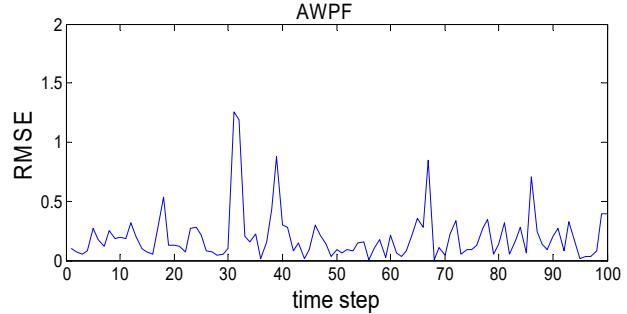

b)

Fig. 5. RMSE curve of general PF and AWPF 
Table 3. Comparison of simulation

\begin{tabular}{|c|c|c|}
\hline & Mean accuracy & Available samples \\
\hline PF & 0.568 & 192 \\
\hline AWPF & 0.292 & 405 \\
\hline
\end{tabular}

\section{Application to Fault Detection}

\subsection{Experimental System}

In this section, the performances of AWPF are verified via fault detection of a rolling bearing. In order to better interpretation of the performances of AWPF, WT method is introduced for comparison.

Fig. 6 shows the experimental platform for fault detection and diagnosis of a rolling bearing. As shown in Fig. 6, the power of the experimental platform is provided by a servo motor (Mitsubishi HG-SR352BJ), with output power $3.5 \mathrm{~kW}$, rated speed $3000 \mathrm{rpm}$, rated voltage $380 \mathrm{~V}$. The loading equipment (Shizuoka RCS2-RA13R) with $300 \mathrm{~kg}$ load is used to exert pressure on the rotating shaft. Two types of rolling bearing (N205 and NU205) are used for obtaining the vibration data of rolling bearing in each state. N205 that outer ring can be disassembled is used for normal, outer-race defect and roller element defect test. NU205 that inner-race can be disassembled is used for inner-race defect state. Fig. 7 shows the three kind of bearing faults, which are artificially processed by using Wire cutting machine, and fault width is $0.3 \mathrm{~mm}$, and fault depth is $0.025 \mathrm{~mm}$.

In present work, data acquisition system includes an accelerometer, signal conditioner, signal recorder and computer. The raw vibration signals are measured via the accelerometer at constant speed $1000 \mathrm{rpm}$, with $50 \mathrm{~Hz}$ sampling frequency. The sapling time is about $20 \mathrm{~s}$. After amplified by the signal conditioner, the signals are transferred into the signal recorder. The raw signals measured in each state are shown in Fig. 8.

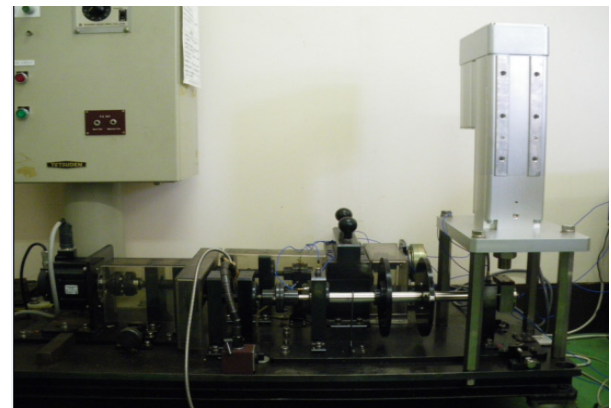

a)

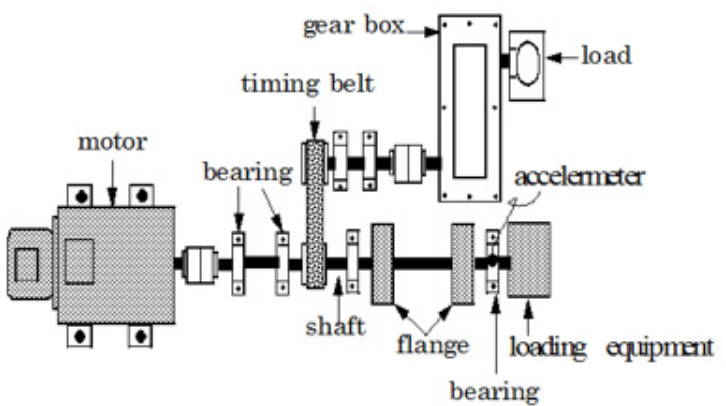

b)

Fig. 6. Illustrate of the experimental setup for test
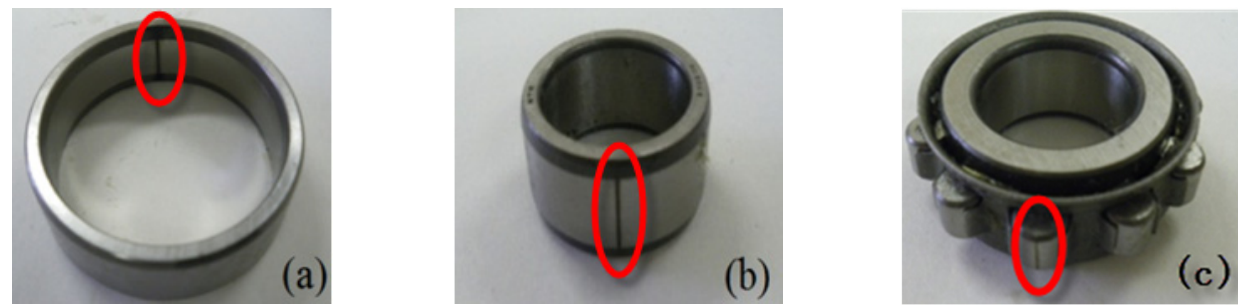

Fig. 7. Bearing defects: a) outer race defect, b) inner race defect, c) rolling defect 


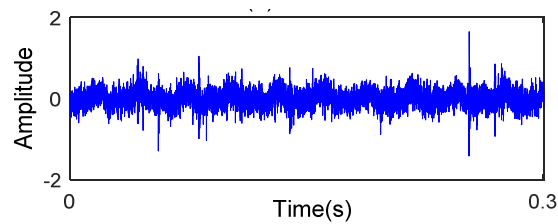

a) Normal

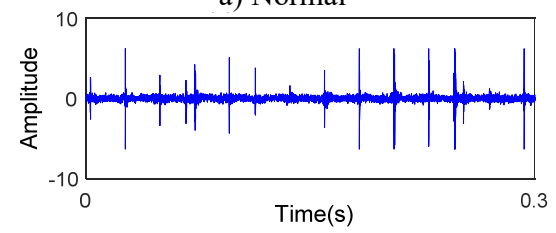

c) Inner-race defect

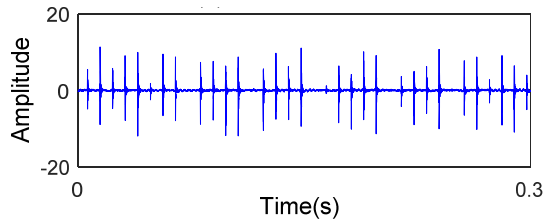

b) Outer-race defect

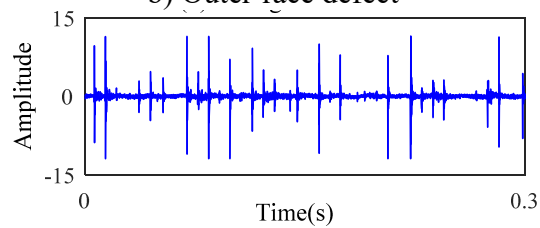

d) Rolling element defect

Fig. 8. Raw vibration signals of the bearing

\subsection{State-space model of vibration signal}

\subsubsection{State Equation}

To use PF technique for FDD, the state model and measurement model described in Eqs. (1) and (2) need to be established firstly. Due to the complexity of mechanism and the diversity of mechanical failures, it is difficult to construct accurate vibration signal model. Thus, time-varying auto regressive (TVAR) method is used to construct the vibration signal model in this paper. Assuming $x_{t}$ is vibration signal, the TVAR model of vibration signal is as follows:

$x_{t}=-\sum_{i=1}^{p} a_{i}(t) x_{t-i}+e_{t}$,

where, $a_{i}$ indicates time-varying coefficient; $p$ denotes model order; $e_{t}$ is residual error of the model:

$a_{i}(t)=-\sum_{j=0}^{m} a_{i j} g_{j}(t)$

where, $m$ is extended dimension; $a_{i j}$ is weight of basic function; $g_{j}(t)$ express time basic function. According to the vibration signal characteristic of rolling bearing, this study choice DCT basic function shown in Eq. (23) to estimate time-varying coefficient of TVAR model:

$g_{j}(t)=a(t) \cos \left(\frac{j \pi(2 k+1)}{2 N}\right)$,

$a(t)= \begin{cases}\sqrt{1 / N}, & t=0 \\ \sqrt{2 / N}, & t \neq 0\end{cases}$

Define:

$A^{T}=\left[a_{10}, a_{11} \ldots a_{1 m} \ldots a_{p 0}, a_{p 1} \ldots a_{p m}\right]$
$X_{t}{ }^{T}=\left[x_{t-1} g_{0}(t) \ldots x_{t-1} g_{m}(t) \ldots x_{t-p} g_{0}(t) \ldots x_{t-p} g_{m}(t)\right]$.

According to least square principle, Eq. (21) can be rewritten as: 
$x_{t}=-X_{t}^{T} A+e_{t}$

Thus, the least square estimates of $A$ is:

$\hat{A}=-\left(\sum_{t=p+1}^{N} X_{t} X_{t}^{T}\right)^{-1} \sum_{t=p+1}^{N} X_{t} x_{t}$

The least square estimates of the variance of $e_{t}$ :

$\hat{\sigma}^{2}=\frac{1}{N-p} \sum_{t=p+1}^{N}\left[x_{t}+\sum_{i=1}^{p} a_{i}(t) x_{t-i}\right]^{2}$.

In this paper, Akaike Information Criterion (AIC) method is introduced for determining model order $p$, and mathematical expression of AIC is given as follows. A detailed description about AIC method can be found in reference [28]:

$\operatorname{AIC}(p)=\ln \hat{\sigma}^{2}+\frac{2 p}{N-r}$

where, $N$ is the length of signal; $r$ indicates pre-order of the model.

\subsubsection{Measurement equation}

Firstly, the original vibration signals measured by the accelerometer are decomposed into 5 layers by using WT with db1 wavelet function. Secondly, the threshold is extracted by ddencpm function. The wavelet coefficients that less than the threshold is retained, and the wavelet coefficients that greater than the threshold value are removed. Lastly, the remaining wavelet coefficients are reconstructed by the waverec function, and the reconstructed signal is the measurement noise.

Measurement equation:

$z_{t}=x_{t}+w_{t}$

where, $x_{t}$ and $z_{t}$ express state and measurement values at time $t$, respectively; $w_{t}$ is measurement noise obtained by WT.

\subsection{Calculation of characteristic frequency}

Condition diagnosis of roller element bearing depends largely on analysing the vibration signals generated by localized defects. Fault types can be identified base on the characteristic frequencies which estimate by the geometry parameters of roller element bearing [29]. The characteristic frequencies of bearing defects can be calculated by the theoretical equation [30].

The characteristic frequencies of the outer race $\left(f_{O}\right)$ :

$f_{o}=\frac{1}{2} n f_{s}\left(1-\frac{D_{B}}{D_{P}} \cos \theta\right)$

The characteristic frequencies of the inner race $\left(f_{I}\right)$ : 
$f_{I}=\frac{1}{2} n f_{s}\left(1+\frac{D_{B}}{D_{P}} \cos \theta\right)$

The characteristic frequencies of the roller $\left(f_{E}\right)$ :

$f_{E}=\frac{D_{P}}{D_{B}} f_{s}\left(1-\frac{D_{B}^{2}}{D_{P}^{2}} \cos \theta\right)$

where $n$ is the number of balls in rolling elements bearing, $f_{r}$ indicates rotating frequency of motor, $D_{B}$ and $D_{P}$ are the ball diameter and the pitch circle diameter, respectively. $\theta$ represents the bearing contact angle of roller bearing.

Above the characteristic frequencies are based on the ideal condition. However, in practice, some sliding motion may occur, which causes slight deviation of the characteristic frequency locations. In this work, the characteristic frequencies of the outer race defect, the inner race defect and the roller element defect are $88.76 \mathrm{~Hz}, 128.3 \mathrm{~Hz}$, and $94.8 \mathrm{~Hz}$, respectively.

\subsection{Fault detection by the proposed method}

Procedure of fault detection by AWPF is given as follows. Firstly, the vibration signals in each state are measured. Secondly, state-space models of the vibration signal are set up by the method described in Section 4.2, and AWPF is used to estimate the probability density function of the system to achieve the purpose of vibration signal denoising. Thirdly, the envelope spectrum information waves in each state were obtained. Finally, we detect the roller bearing state by the extracted spectra. Figs. 9-11 show parts of the verification results.

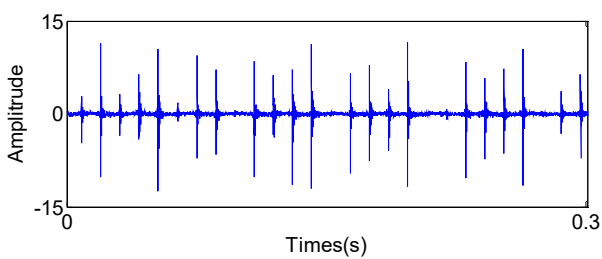

a) In time domain

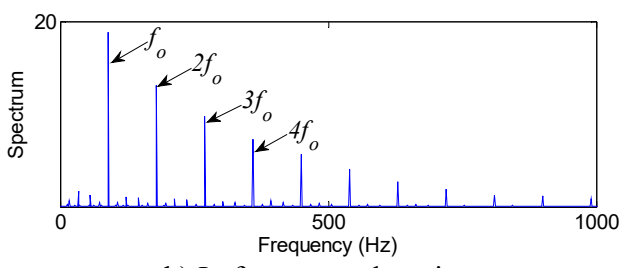

b) In frequency domain

Fig. 9. Signal of the out-race defect after AWPF

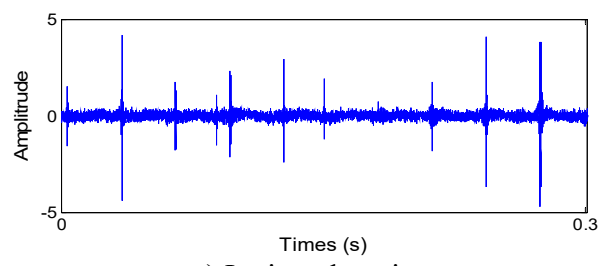

a) In time domain

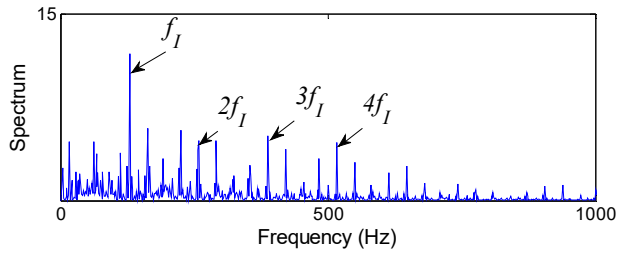

b) In frequency domain

Fig. 10. Signal of the Inner race defect after AWPF

From Fig. 9(b), the impact repetition frequency $f_{O}$ is $89.8 \mathrm{~Hz}$ and its harmonics $2 f_{O}, 3 f_{O}$ and $4 f_{O}$ are about $180.1 \mathrm{~Hz}, 270.2 \mathrm{~Hz}$ and $360.1 \mathrm{~Hz}$, respectively. $f_{O}$ approaches the outer race defect characteristic frequency at $88.7 \mathrm{~Hz}$ that calculated by Eq. (20); therefore, we judge the fault type as the outer race defect. Similarly, in Fig. 10(b) the impact repetition frequency $f_{I}$ is about $129.7 \mathrm{~Hz}$ and its harmonics $2 f_{I}, 3 f_{I}$ and $4 f_{I}$ are about $259.4 \mathrm{~Hz}, 387.6 \mathrm{~Hz}$ and $517.3 \mathrm{~Hz}$, respectively. $f_{I}$ approaches the Inner race defect characteristic frequency at $128.3 \mathrm{~Hz}$ that calculated by Eq. (21); therefore, it was identified as the inner race defect by these spectra. On the basis of Fig. 11(b), the characteristic frequency $f_{E}$ is about $96.1 \mathrm{~Hz}$, and close to the rolling element defect pass-frequency at $94.8 \mathrm{~Hz}$ that calculated by Eq. (22); its harmonics $2 f_{E}$ is about 
192.5 Hz. Although there are not any obviously characteristic at harmonics $3 f_{E}$ and $4 f_{E}$, the rolling element defect of the bearing can be identified by the characteristic frequency $f_{E}$ and $2 f_{E}$.

As shown in Figs. 10 and 11, the repetition of harmonics and sidebands throughout the spectra of the inner race defect and the rolling element defect, which make the inner race and the roller defects are not easy to identify as the outer race defect. However, those defects can be identified by the corresponding bearing characteristic frequencies.

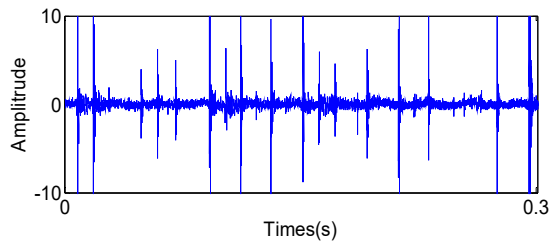

a) In time domain

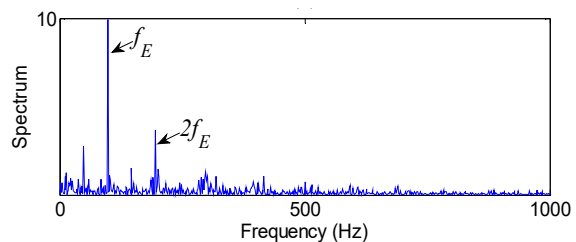

b) In frequency domain

Fig. 11. Signal of the roller element defect after AWPF

\subsection{Fault diagnosis by traditional PF}

In order to verify the effectiveness of the proposed method, several popular signal processing methods such as traditional PF, WT and Empirical Mode Decomposition (EMD) are used to compare with the proposed method in this paper.

Figs. 12-14 show the diagnosis results by using traditional PF. From the Fig. 12 the impact repetition frequencies are $90.1 \mathrm{~Hz}, 180.1 \mathrm{~Hz}$ and $270 \mathrm{~Hz}$ respectively, and close to the characteristic frequency of the outer race defect and its harmonics. Hence, it was identified as the outer race defect. However, as shown in the Figs. 13 and 14, it is difficult to find the fault characteristic frequencies of inner race defect and rolling element defect.

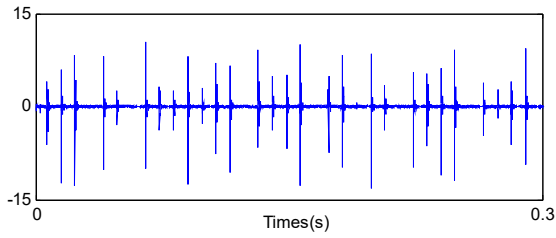

a) In time domain

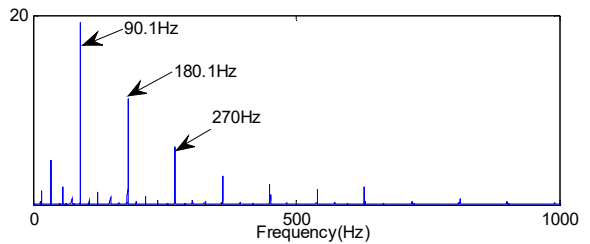

b) In frequency domain

Fig. 12. Signal of the out-race defect after traditional PF

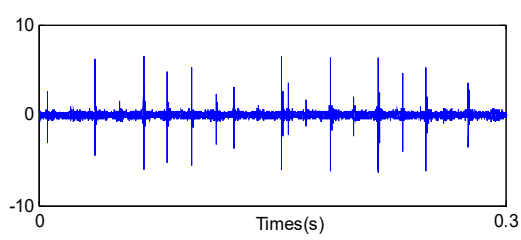

a) In time domain

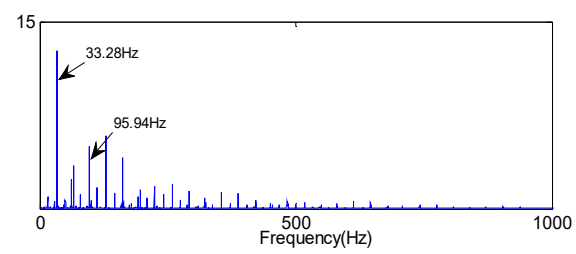

b) In frequency domain

Fig. 13. Signal of the inner race defect after traditional PF

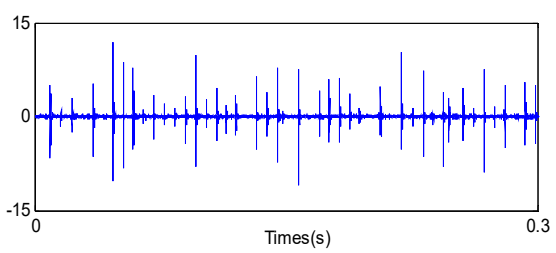

a) In time domain

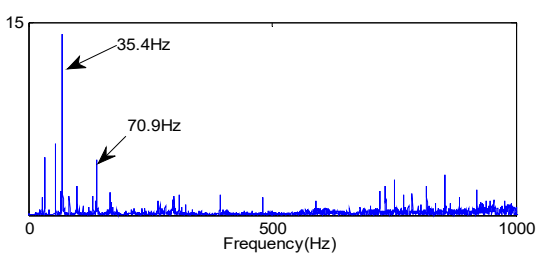

b) In frequency domain

Fig. 14. Signal of the roller element defect after traditional PF 


\subsection{Fault diagnosis by wavelet transform}

WT is a time-frequency signal analysis method, which can divide signal into different frequency regions and carry out signal decomposition and information extraction [31].

In this work, the wavelet function ReverseBior 2.8 is chosen for fault diagnosis, and diagnosis flowchart is as follows. Firstly, the raw signal measured in each defect state is decomposed into four levels in low-frequency regions $(0-25 \mathrm{kHz}, 0-12.5 \mathrm{kHz}, 0-6.25 \mathrm{kHz}$ and $0-3.125 \mathrm{kHz})$ and high-frequency regions $(25-50 \mathrm{kHz}, 12.5-25 \mathrm{kHz}, 6.25-12.5 \mathrm{kHz}$ and $3.125-6.25 \mathrm{kHz})$ by ReverseBior 2.8. Secondly, the signal components at each level are reconstructed in the time domain by reconstruction function. Lastly, the envelope spectra at each level are obtained by the FFT technique. The vibration caused by bearing defect is appeared in the high-frequency region generally. Hence, in present work we only analysis the signals in high-frequency levels. The envelope spectra of each high-frequency level are shown in Figs. 15-18. From the Figs. 15-18(b) the impact repetition frequency approaches to the twice outer race pass-frequency at about $180 \mathrm{~Hz}$, they approach the twice outer race characteristic frequency $178 \mathrm{~Hz}$, hence, it was identified as the outer race defect. However, as shown in the Figs. 15-18(c) and (d), it is difficult to find the fault characteristic frequencies of inner race defect and rolling element defect at each level, because the signal contains strong noise, the fault characteristic frequencies caused by the defective bearing and its harmonics are buried.

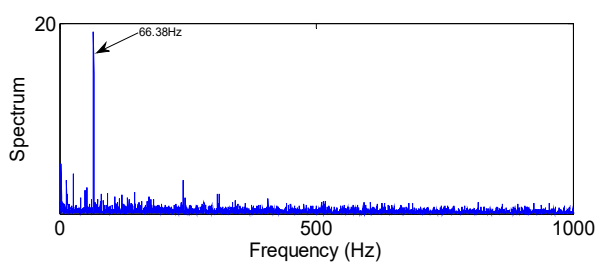

a) Normal

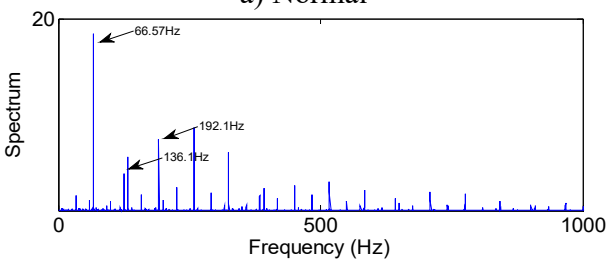

c) Inner-race defect

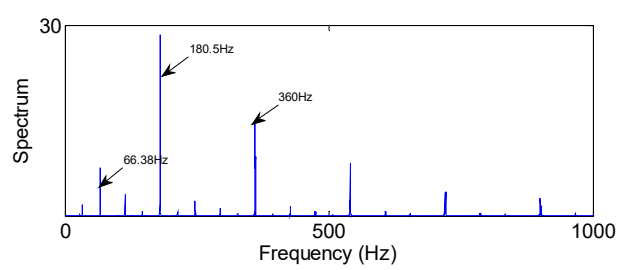

b) Outer-race defect

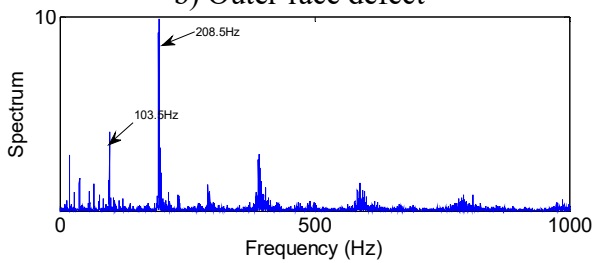

d) Rolling element defect

Fig. 15. Envelope spectra in $25-50 \mathrm{kHz}$ level

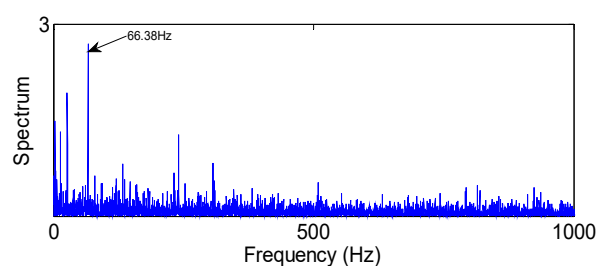

a) Normal

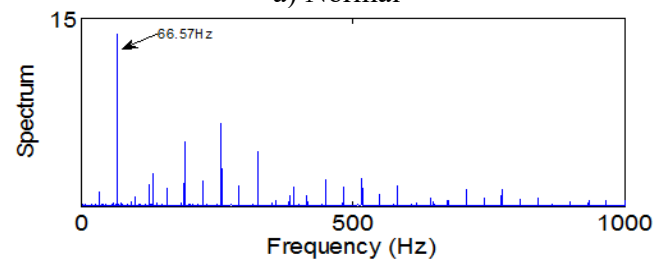

c) Inner-race defect

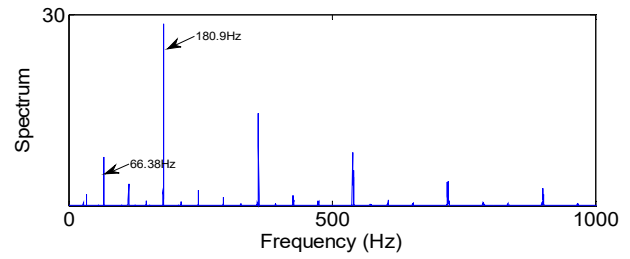

b) Outer-race defect

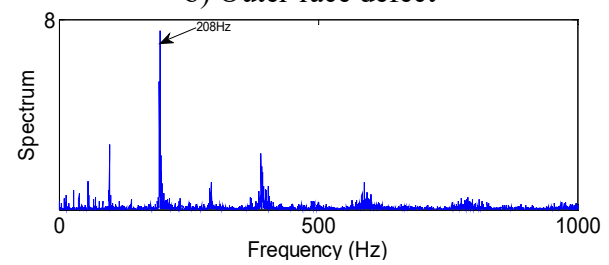

d) Rolling element defect

Fig. 16. Envelope spectra in $12.5-25 \mathrm{kHz}$ level 


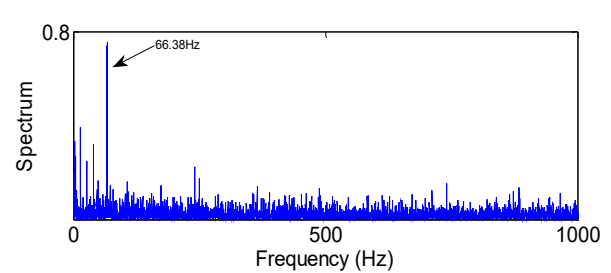

a) Normal

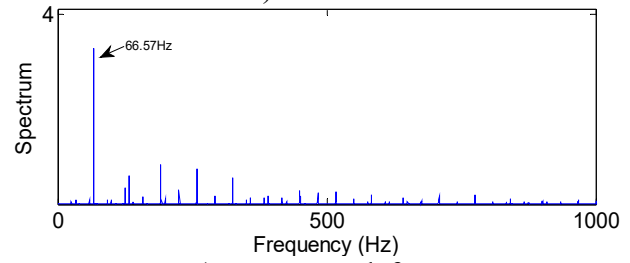

c) Inner-race defect

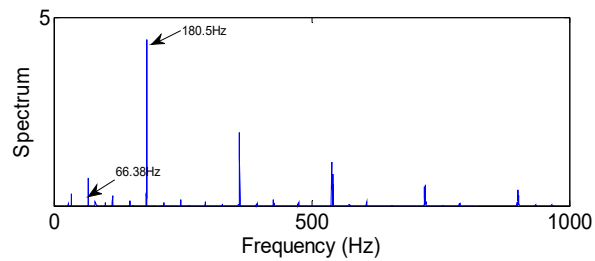

b) Outer-race defect

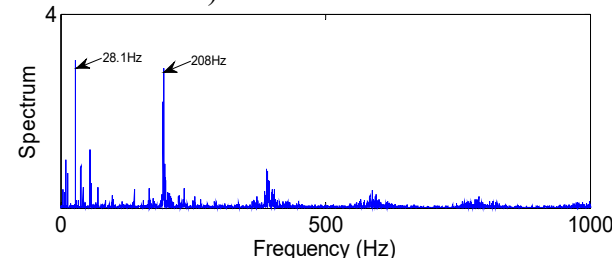

d) Rolling element defect

Fig. 17. Envelope spectra in $6.25-12.5 \mathrm{kHz}$ level

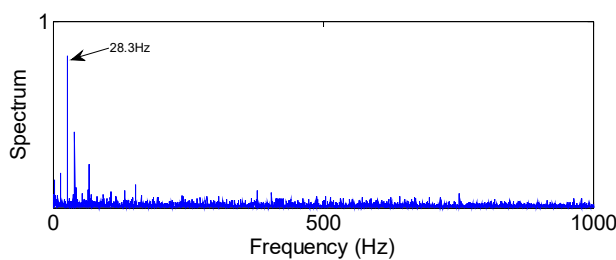

a) Normal

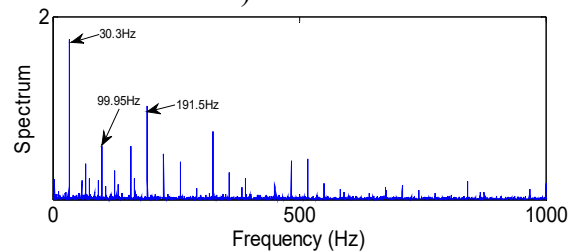

c) Inner-race defect

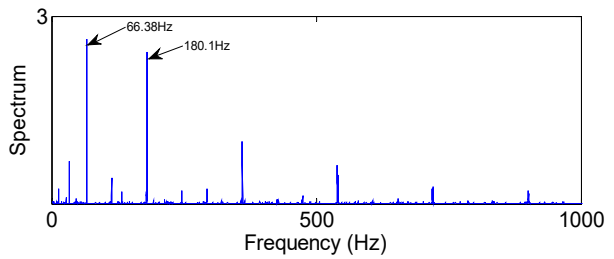

b) Outer-race defect

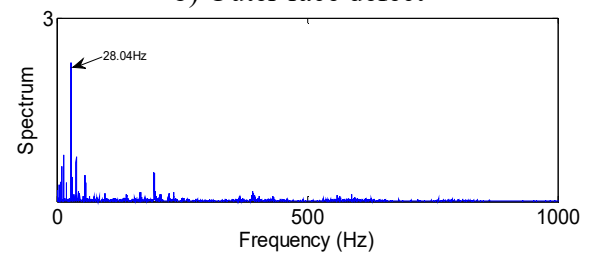

d) Rolling element defect

Fig. 18. Envelope spectra in $3.125-6.25 \mathrm{kHz}$ level

\subsection{Fault diagnosis by EMD}

EMD is a non-stationary signal adaptive decomposition method proposed by Huang. According to the characteristics of the signal, EMD can adaptively select the frequency band, and determine the signal in different frequency resolution. In the last 10 years EMD has made great progress and has been successfully applied in mechanical fault diagnosis [32]. In this work, we also compare the proposed method with EMD method, and diagnosis procedure is as follows. Firstly, the vibration signal that measured in each state is decomposed into the different characteristic scale intrinsic mode function (IMF) by EMD. Secondly, the envelope spectra of each IMF are obtained by the FFT technique. Finally, the condition and fault type of the bearing are determined by analyzing envelope spectra of each IMF. As the high-frequency signal is distributed on the first few IMF components, in present work we only analysis the first four IMF components. The envelope spectra of IMFs in each state are shown in Figs. 19-21.

From Fig. 19(a), we can find the impact repetition frequency at about $89.3 \mathrm{~Hz}$ and $180.1 \mathrm{~Hz}$, which approaches to the outer race defect characteristic frequency and the twice outer race defect characteristic frequency. Therefore, we judge the fault type as the outer race defect. Similarly, in Fig. 20(c) the impact repetition frequency at about $130.2 \mathrm{~Hz}$ can be find, and it approaches the Inner race defect characteristic frequency. Therefore, it was identified as the inner race defect by this spectrum. However, in Fig. 21 it is difficult to find the fault characteristic frequencies of the rolling element defect at each IMF. 


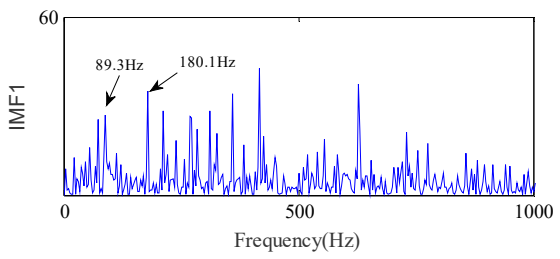

a)

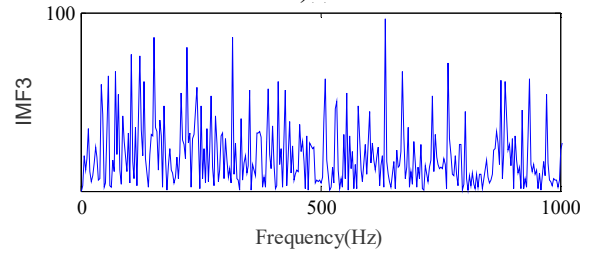

c)

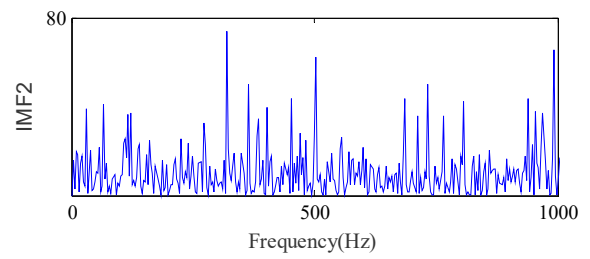

b)

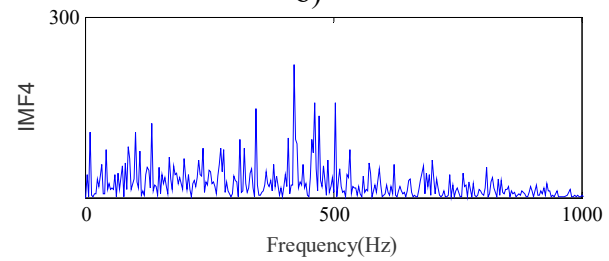

d)

Fig. 19. Signal of the out-race defect after EMD

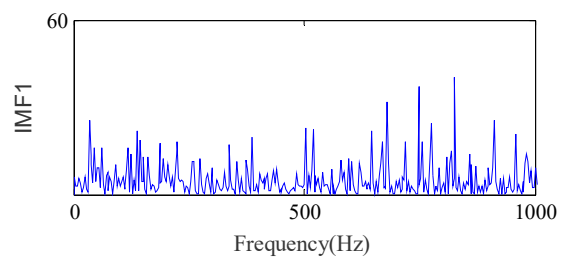

a)

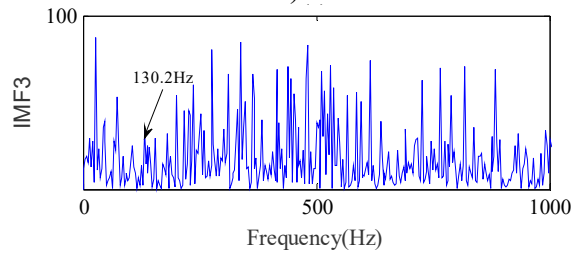

c)

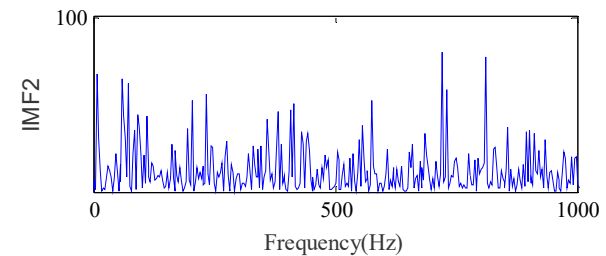

b)

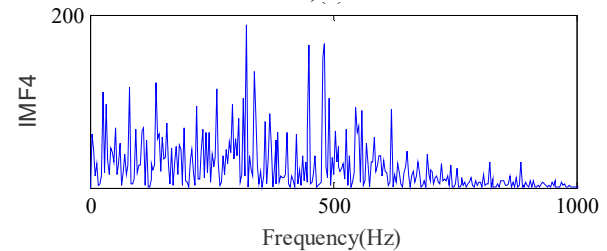

d)

Fig. 20. Signal of the Inner race defect after EMD

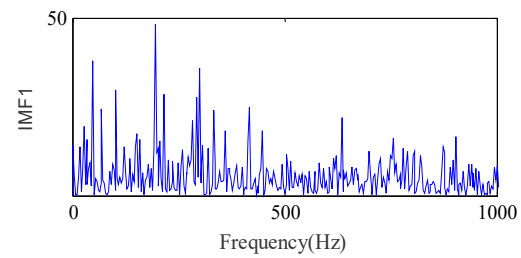

a)

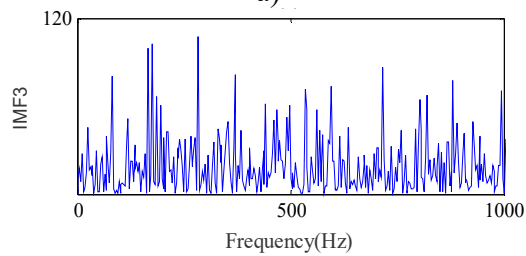

c)

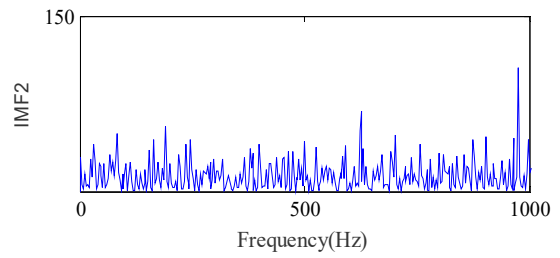

b)

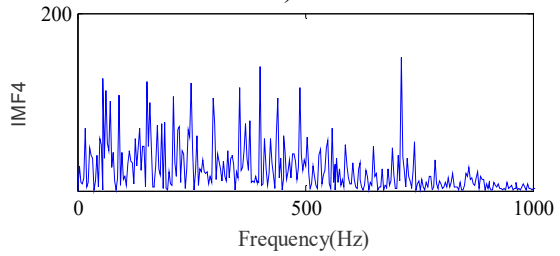

d)

Fig. 21. Signal of the roller element defect after EMD

On the basis of above verification results, three types bearing defects were effectively and accurately identified via the method proposed in this study. This shows that the method proposed in this study is the most efficacious for diagnosing bearing faults and eliminate much noise from 
the signal. Although the outer race defect of the bearing can be identified by using WT and traditional PF, it is difficult to identify the inner race and roller element defects. In add cation, the outer race and inner defects of the bearing can be identified with EMD method. However, the roller element defect cannot be identified, because the signal contains strong noise, the fault characteristic frequencies caused by the defective bearing and its harmonics are buried.

Ke Li, Peng Chen and Jingjing Wu conceived and designed the experiments; Ke Li and Peng Chen performed the experiments; Ke Li, Yiya Liu and Su Lei analyzed the data; Ke Li wrote the paper.

\section{Conclusions}

In order to effectively monitor condition and detect fault types of high nonlinear system, and extract the features of system state under strong noise background, this paper proposed a novel FDD method based on the modified PF which called AWPF. The BPNN was incorporated in PF for adaptively adjusting weight of particle. In AWPF, the large weight particles were split into two small weight particles, the particles with smaller weight was adjusted by using artificial neural network. By which the particles in the low probability density region were adjusted to the high probability density region, and particle diversity was increased. The problem of particle leanness was solved effectively. Moreover, TVAR and AIC methods were presented to establish state space model for state estimation. Finally, the proposed method was implemented for fault diagnosis on a roller bearing. We also compared the proposed method with traditional PF, WT and EMD techniques. The verification results shown that three types bearing fault had been effectively identified by the proposed method. However, these bearing faults were difficult to detect using traditional PF, WT and EMD techniques.

\section{Acknowledgements}

The authors would like to acknowledge the financial support of the National Key Technology Support Program of China (2015BAF16B02), Fundamental Research Funds for the Central Universities (Grant No. JUSRP51732B and JUSRP51511).

\section{References}

[1] Jing L., Liangsheng Q. Feature extraction based on Morlet wavelet and its application for mechanical fault diagnosis. Journal of Sound and Vibration, Vol. 234, Issue 1, 2000, p. 135-148.

[2] Chen P., Toyota T., He Z. Automated function generation of symptom parameters and application to fault diagnosis of machinery under variable operation-conditions. IEEE Transactions on Systems, Man, and Cybernetics - Part A: Systems and Humans, Vol. 31, Issue 6, 2001, p. 775-781.

[3] Li K., Chen P., Wang S. M. An intelligent diagnosis method for rotating machinery using least squares mapping and a fuzzy neural network. Sensors, Vol. 5, 2012, p. 5919-5939.

[4] Pacas M., Villwock S., Dietrich R. Bearing damage detection in permanent magnet synchronous machines. Energy Conversion Congress and Exposition, Vol. 9, 2009, p. 1098-1103.

[5] Cheng J. S., Yu D. J., Yang Y. Application of an impulse response wavelet to fault diagnosis of rolling bearings. Mechanical Systems and Signal Processing, Vol. 21, Issue 2, 2007, p. 920-929.

[6] Randall R. B., Antoni J. Rolling element bearing diagnostics - a tutorial. Mechanical Systems and Signal Processing, Vol. 25, Issue 2, 2011, p. 485-520.

[7] Bianchini C., Immovilli C., Cocconcelli M., Rubiniand R., Bellini A. Fault detection of linear bearings in brushless AC linear motors by vibration analysis. IEEE Transactions on Industrial Electronics, Vol. 58, Issue 5, 2011, p. 1684-1694.

[8] Stack J. R., Habetler T. G., Harley R. G. An amplitude modulation detector for fault diagnosis in rolling element bearing. IEEE Transactions on Industrial Electronics, Vol. 51, Issue 5, 2004, p. 1097-1102.

[9] Rajagopalan S., Restrepo J. A., Aller J. M., Habetler T. G., Harley R. G. Nonstationary motor fault detection using recent quadratic time-frequency representations. IEEE Transactions on Industry Applications, Vol. 44, Issue 3, 2008, p. 735-744. 
[10] Randall R. B, Antoni J. Rolling element bearing diagnostics - a tutorial. Mechanical Systems and Signal Processing, Vol. 25, 2011, p. 485-520.

[11] Sheen Y. T. An analysis method for the vibration signal with amplitude modulation in a bearing system. Journal of Sound and Vibration, Vol. 303, Issue 3, 2007, p. 538-552.

[12] Mori K., Kasashima N., Yoshioka T., Ueno Y. Prediction of spalling on ball bearing by applying the discrete wavelet transform to vibration signal. Wear, Vol. 195, 1996, p. 162-168.

[13] Hu Q., He Z. J., Zhang Z. S., Zi Y. Y. Fault diagnosis of rotating machinery based on improved wavelet package transform and SVM ensemble. Mechanical Systems and Signal Processing, Vol. 21, Issue 2, 2007, p. 688-705.

[14] Wang Z. H., Shen Y., Zhang X. L. Attitude sensor fault diagnosis based on Kalman filter of discrete-time descriptor system. Journal of Systems Engineering and Electronics, Vol. 23, Issue 6, 2012, p. 914-920.

[15] Yang S. K. An experiment of state estimation for predictive maintenance using Kalman filter on a DC-motor. Reliability Engineering and System Safety, Vol. 75, Issue 1, 2002, p. 103-111.

[16] Ozbek L., Efe M. An adaptive extended Kalman filter with application to compartment models. communication in statistics-simulation and computation. Communications in Statistics - Simulation and Computation, Vol. 33, Issue 1, 2004, p. 145-158.

[17] Dias S. S., Bruno M. G. Cooperative target tracking using decentralized particle filtering and RSS sensors. IEEE Transactions on Signal Processing, Vol. 61, Issue 14, 2013, p. 3632-3646.

[18] Hiremath S. A., Van Der Heijden G. W., van Evert F. K., Stein A., Ter Braak C. J. Laser range finder model for autonomous navigation of a robot in a maize field using a particle filter. Computers and Electronics in Agriculture, Vol. 100, 2014, p. 41-50.

[19] Wei T., Huang Y. F., Philip Chen C. L. Adaptive sensor fault detection and identification using particle filter algorithms. IEEE Transactions on Systems, Man, and Cybernetics, Vol. 39, Issue 2, 2009, p. 201-213.

[20] Yin S., Zhu X. P. Intelligent particle filter and its application to fault detection of nonlinear system. IEEE Transactions on Industrial Electronics, Vol. 62, Issue 6, 2015, p. 3852-3861.

[21] Liu Y., Sun D. Q., Kong L. Rao-blackwellized particle filtering for fault detection and diagnosis. Proceedings of the 29th Chinese Control Conference, 2010, p. 29-31.

[22] Saha B., Goebel K., Poll S., Christophersen J. Prognostics methods for battery health monitoring using a Bayesian framework. IEEE Transactions on Instrumentation and Measurement, Vol. 58, Issue 2, 2009, p. 291-296.

[23] Aesarendra W. C, Niu G., Yang B. Machine condition prognosis based on sequential Monte Carlo method. Expert Systems with Applications, Vol. 37, Issue 3, 2010, p. 2412-2420.

[24] Doucet A., de Freitas N., Gordon N. Sequential Monte-Carlo Methods in Practice, Springer. New York, 2001.

[25] Douc R., Cappé O. Comparison of resampling schemes for particle filtering. Proceedings of the 4th International Symposium on Image and Signal Processing and Analysis, 2005, p. 64-69.

[26] Arulampalam M. S., Maskell S., Gordon N., Clapp T. A tutorial on particle filters for online nonlinear/non-Gaussian Bayesian tracking. IEEE Transactions on Signal Processing, Vol. 50, Issue 2, 2002, p. 174-188.

[27] Buscema M. Back propagation neural network. Substance Use and Misuse, Vol. 33, Issue 2, 1998, p. 233-70.

[28] Fei W. C., Bai L. Time-varying parameter auto-regressive models for autocovariance nonstationary time series. Science in China Series A: Mathematics, Vol. 52, Issue 3, 2009, p. 577-584.

[29] Tandon N., Choudhury A. A review of vibration and acoustic measurement methods for the detection of defects in rolling element bearings. Iribology International, Vol. 320, 1999, p. 469-480.

[30] Chen P., Toyota T. Sequential fuzzy diagnosis for plant machinery. JSME International Journal Series C, Vol. 46, 2003, p. 1121-1129.

[31] Li K., Chen P., Wang H. Q. Intelligent diagnosis method for rotating machinery using wavelet transform and ant colony optimization. IEEE Sensors Journal, Vol. 12, Issue 7, 2012, p. 2474-2484.

[32] Li Y., Xu M., Wei Y., Huang W. An improvement EMD method based on the optimized rational Hermite interpolation approach and its application to gear fault diagnosis. Measurement, Vol. 63, 2015, p. 330-345. 

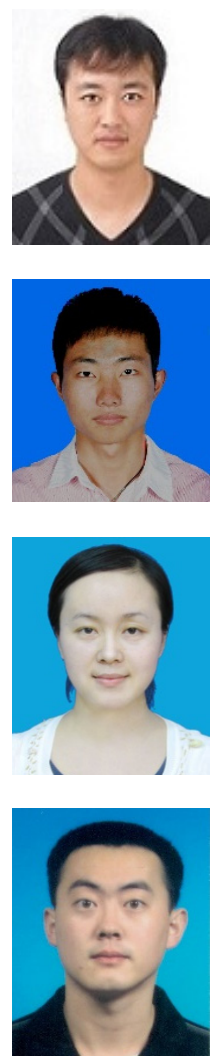

Lei Su received the Ph.D. degree from the Huazhong University, Wuhan, China, in 2015. Currently, he is a lecturer of the College of Mechanical Engineering, Jiangnan University, Wuxi, China. His research interests include non-destructive testing, signal processing and structural health monitoring.

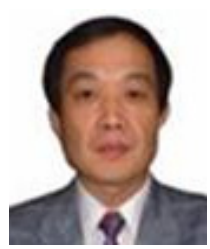

Yiya Liu received his B.S. degree from Anhui Polytechnic University, China in 2014. He is currently a Master in the School of Mechanical Engineering, Jiangnan University, China. His research interests include fault diagnosis of plant machinery and signal processing.

Jingjing Wu received his Ph.D. from Shanghai Jiao Tong University, China in 2012. She is currently an Associate Professor in the School of Mechanical Engineering, Jiangnan University, China. Her research interests are signal processing, Bayesian filtering, pattern recognition, and multisensor data fusion

Peng Chen graduated from the Doctoral course of the Kyushu University, Japan in 1990, and currently a Professor of the Graduate School of Bioresources, Mie University, Japan. His research interest includes condition diagnosis of plant machinery, information and signal processing. 